\title{
Momordica cymbalaria Fruit Extract Using Bio Production of Selenium Nanoparticles and Assessment of Antibacterial Activity
}

\section{Paulraj Suganya*, Sherlin Shruthi Sathia Singh and Sundaramoorthy Pandi}

Department of Biotechnology, Sri Kaliswari College (Autonomous), Sivakasi, Tamil Nadu, India

*Corresponding Author: Paulraj Suganya, Department of Biotechnology, Sri Kaliswari College (Autonomous), Sivakasi, Tamil Nadu, India.
Received: September 06, 2021

Published: November 10, 2021

(C) All rights are reserved by Paulraj Suganya., et al.

\begin{abstract}
Synthesis of nano- selenium was achieved by fruit extract. This aim of the present study is bioproduction of selenium nanoparticles using fruit extract which was from Momordica cymbalaria (athalkkai in Tamil). The confirmation of selenium nanoparticles was confirmed by Transmission Electron Microscope (TEM), Scanning Electron Microscope (SEM), X-Ray Crystallography (XRD), Fourier Transform Infrared Spectroscopy (FTIR), UV visible spectroscopy (UV - Vis) and Se NPs were examined the antibacterial activity of the biosynthesized Se NPs. The overall result from this study clearly indicates that the biosynthesized of Se NPs may have a great potential for further use.
\end{abstract}

Keywords: Momordica cymbalaria; Athalakkai; Selenium Nanoparticles; Antibacterial Activity

\section{Introduction}

Momordica cymbalaria is an ancient plant along with the cucurbitaceous family and also a medicinal plant. It is a vegetables crop and grows at rainy sessions. The Momordica cymbalaria plants found in Tamil Nadu, Karnataka, Madhya Pradesh, Maharashtra and Andra Pradesh [14].

The biosynthesis of nanoparticles offers benefits of cost effective, eco-friendly, useless energy and can provide nanoparticles with better defined size and morphology of nanoaprticles [11]. The Momordica cymbalaria edible parts of this species are the fruits, its contain rich nutritional value such as calcium, potassium, Vitamin and fibers [18]. These plant parts were traditionally used as a skin diseases, ulcer, diabetic, tonic, stomach ache, diarrhea and abortion by the south Indian treatments [22].
Nanomaterials are new, unique and size of the particles was 1-100 $\mathrm{nm}$, they have physical chemical properties to compare the bulk material [6]. Green synthesis principally concerns eliminating unsafe waste, and utilizing property processes, implementing environmentally friendly chemicals, solvents, and renewable materials [1].

Now a day's plant mediated synthesis become popular to be biosynthesised nanoparticles in which are uniform and stable in nature $[12,15]$. In green-nanotechnology, varied metal ions nanoparticle synthesis have been reported from biological extracts viz., yeast, fungi, bacteria, algae, plant and animal system, etc. The plant extracts and microbes were found to contain biomolecules that act as capping and reducing agents. Plant extracts based reduction strategies are found significant as simple and cost-effec- 
tive for synthesizing metal ion nanoparticles under delicate experimental conditions like comparatively low reaction temperature and ambient pressure [9]. The aim of this present study is synthesis and characterization of Se NPs and also to assess their antibacterial activity against the humab bacterial pathogens.

\section{Materials and Methods}

Preparation of plant extracts

M. cymbalaria plant fruits were collected from local area of Virudhunagar district, Tamil Nadu, India. The collected fruit samples were washed with tap water to removing the impurities of fruits and surface sterilized with sodium hypochloride for 2 mins. The fresh fruits $(10 \mathrm{~g})$ were crushed with using mortar and pestle. The collected extract was filtered and stored for further using of studies.

Synthesis of selenium nanoparticles

Sodium selenite $\left(\mathrm{Na}_{2} \mathrm{SeO}_{3}\right)$ solution was prepared by double distilled water and mixed by filtrate of $M$. cymbalaria fruits extract and incubated at $10 \mathrm{hrs}$ in room temperature. The color change due to reaction in the solution was observed and recorded. The synthesized Se NPs was later centrifuged at 10,000 rpm for 20 mins to collect the Se NPs pellets for further studies.

\section{Characterization of nanoparticles}

Transmission electron microscope (TEM)

The biosynthesized of Se NPs was analysed TEM performed on a CM 200 model Philips at an accelerating voltage of 20-200kV. A drop of Se NPs reveals that the shape and size of the synthesized nanoparticles.

\section{Scanning electron microscope (SEM)}

The shape and morphology of Se NPs were analyzed using the Scanning Electron Microscope (SEM) (Vega 5 TESCAN 129ev). In SEM analysis, Se NPs NPs spread uniformly on the SEM plate were placed acetone clean carbon tape and applied sputter coat. The sample plate was followed in a vacuum chamber for 1 hour and loaded in an SEM instrument. The energy Dispersive X-ray Spectrum (EDAX) recorded the areas of the solid surface of manganese oxide nanoparticles to reveal the chemical composition of nanoparticles. The EDAX were done by Bruker, nano D-12480 instrument.
X-ray diffraction analysis (XRD)

The biosynthesized of Se NPs was coated on the glass slides were measured by using an X-ray diffractometer model (XRD6000 , Shimadzu, Japan) with determining the crystallinity of Se nanoparticles.

\section{Fourier transform infrared spectroscopy (FTIR)}

The biosynthesized of Se NPs were FT-IR spectral measurements in the range of $400-4000 \mathrm{Cm}^{-1}$ to identify the biomolecules responsible for the reduction, capping, and stabilizing of nanoparticles during their synthesis.

\section{UV visible spectroscopy (UV-Vis)}

The biosynthesized of Se NPs solution was collected and analysis was carried out by using UV- Vis Spectrophotometer at 200-800 nm range.

\section{Antibacterial activity}

Biosynthesized of Se NPs was treated against various pathogenic bacteria by using the agar diffusion method. The tested microorganisms Staphylococcus aureus, Bacillus cereus, Escherichia coli, Pseudomonas aeruginosa and Klebsiella pneumonia. An active bacterial culture was obtained by shifting the culture to the Muller Hinton agar medium. The bacterial strains were uniformly spread onto swabbed the petriplates and each agar plates was created a wells of $8.00 \mathrm{~mm}$ size using gel puncture. Se NPs solution used for various concentrations $(20,40,60,80,100 \mathrm{mg} / \mathrm{ml})$ was prepared and placed into each well on the petri plates. The petri plates were incubated at $37^{\circ} \mathrm{C}$ and the zone of inhibition was measures in millimetre with help of a rule.

\section{Results and Discussion}

\section{Synthesis of nanoparticles}

The extract of $M$. cymbalaria using biosynthesized Se NPs and characterization of nanoparticles is done based on their shape, size and also assessed the biological activity of antibacterial activity under in vitro condition. We have reported the fruit extract of $M$. cymbalaria changed its color from green to light orange with mixed with sodium selenite at room temperature (Figure 1). The previous researchers are reported that the color change is an indication of the formation of Se NPs $[5,19]$. The color change of nanoparticles is 
due to surface plasmon resonance of the metal nanoparticles [23] and regarding the main cause is presence of numerous bioactive compounds and secondary metabolites like phenols, terpenoids, polysaccharides, flavonoids etc [14] for the response of accelerating reaction and capping of Se NPs.
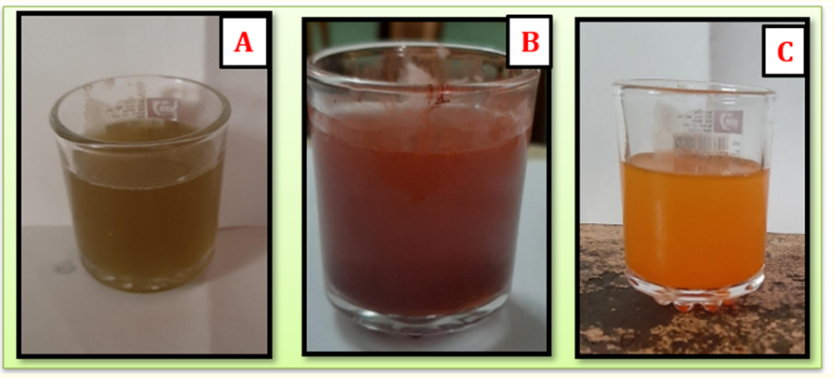

Figure 1: Image showing the color change pattern during nanoparticles synthesis A) Crude fruit extract B) Aqueous solution of Sodium selenite C) Sodium selenite with the fruit extract due to Se NPs formation.

\section{Transmission electron microscope (TEM)}

Size and morphology of Se NPs were identified by this technique. Nano-selenium exhibits spherical shape with an average diameter between 15 - $18 \mathrm{~nm}$. TEM image and its histogram of chemically synthesized Se NPs are illustrated in figures 2 (a) and (b). These images depict that the shape of Se NPs is in the spherical manner which aggress with the SEM analysis result [13] have also described a comparable result where they produced nano-selenium 20-80 nm. Spherically shaped and 44-92 nm sized Se NPs was synthesized by Chen., et al. 2008.
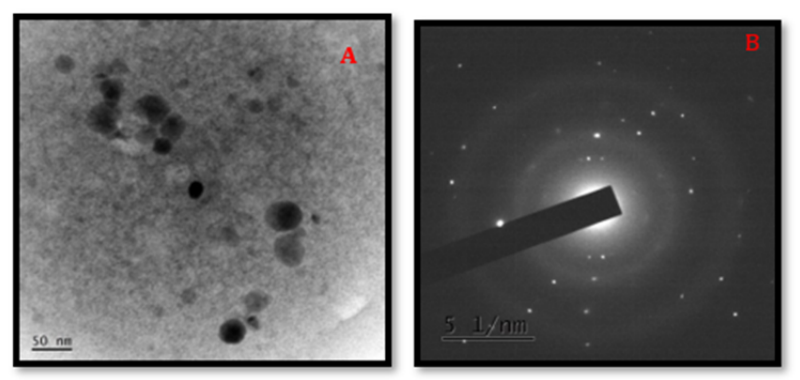

Figure 2: TEM images of synthesized Se NPs using fruit extract of M. cymbalaria.

\section{Scanning electron microscope (SEM)}

SEM is used for studying morphological characteristics of nanoparticles. SEM is capable of imaging pictures with high resolution. Energy dispersive X-ray diffractive (EDAX) is commonly found with SEM instrument which is used to find out the chemical level elemental composition of the samples [21]. SEM image of chemically synthesized Selenium nanoparticles revealed that it is spherical in shape and uniformly distributed (Figure 3(A)). [12] used these techniques for analyzing the morphology and structure of Selenium nanoparticle and produced spherical and smaller sized particle range between $30-100 \mathrm{~nm}$. EDX analysis proves that obtained nanoparticles are pure in nature (Figure 3 (B)). The chemical constituents of the sample were analyzed by Kargar and Razi., et al. 2011 where they could yield about pure selenium.
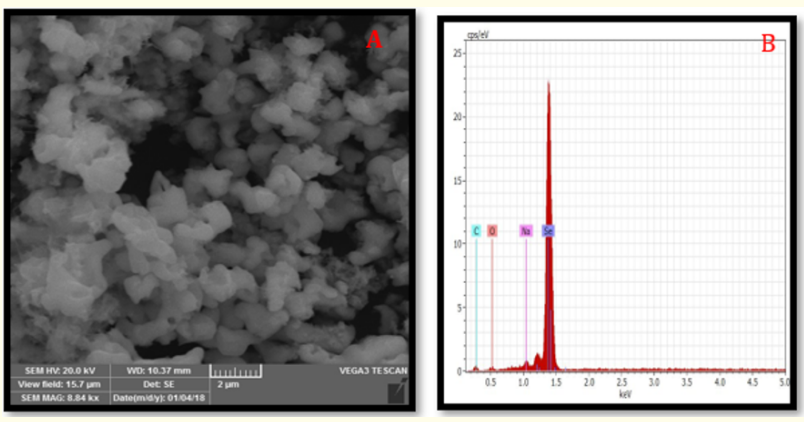

Figure 3: (A) SEM images of synthesized Se NPs using fruit extract of M. cymbalaria (B) EDAX images of synthesized Se NPs using fruit extract of M. cymbalaria.

\section{X-ray diffraction analysis (XRD)}

The crystallite nature of chemically prepared nano-selenium was examined by XRD. This analytical method aids the determination of crystallite materials and it also provides details of unit cell dimensions Dorofeey., et al. 2012 reported that shape and breadth of reflection help to find out substructures in the nanosubstances. Obtained nano-selenium was highly crystalline and all diffraction peaks are well indexed as $23.5616^{\circ}, 29.7572^{\circ}, 41.4821^{\circ}, 43.6615^{\circ}$,

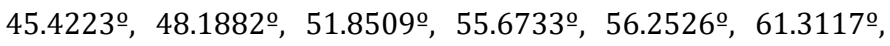
$65.4128^{\circ}, 68.6359^{\circ}$ and $71.4573^{\circ}$ which corresponds to 100,101 , 110, 012, 111, 200, 201, 003, 112,013, 120, 211 and 113 crystal plane respectively which in accords with JCPDS 86-2246 is given in (Figure 4) By adopting Scherrer's equation crystalline nature of nano-selenium was confirmed. 
$\mathrm{D}=0.94 \lambda / \beta \cos \theta$

The calculated crystallite size of nano-selenium was $28 \mathrm{~nm}$. A similar study was also carried out by Khiralla, and El-Deeb. (2015) in which they pointed out sharp peaks from the $2 \theta$ values.

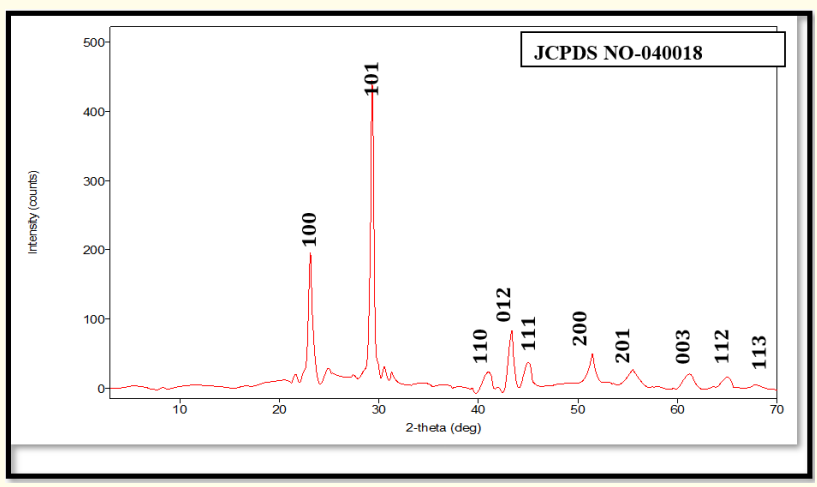

Figure 4: X-ray diffraction pattern of synthesized Se NPs using fruit extract of $M$. cymbalaria.

Fourier transform infrared spectroscopy (FTIR)

FTIR spectra are captured in the frequency ranges from 400 to $4000 \mathrm{~cm}^{-1}$. This frequency absorption helps to identify elements involving in the fabrication of nano-selenium. FT-IR spectra of chemically synthesized $M$. cymabalaria fruit extract and nano-selenium are shown in (Figure $5 \mathrm{~A}$ and B). The stabilized Se NPs were representing 4000-500 $\mathrm{cm}^{-1}$ and determined the various functional groups responsible for reducing and stabilizing Se NPs under the effect of fruit extract. The results revealed that Se NPs is strongly associated with - $\mathrm{OH}$ stretching, $\mathrm{CH} 2$ stretching, and primary amide groups. These functional groups are strong evidence for native protein served as reducing and stabilizing agent of Se NPs nanoparticles, and the absorption peaks are correlated with earlier reports (Mallikarjuna., et al. 2011).

\section{UV visible spectroscopy (UV- Vis)}

UV-Vis spectroscopy results indicate the sharp and strong peaks represents at $370 \mathrm{~nm}$ which is specific for the Se NPs (Figure $6 \mathrm{~A}$ and $\mathrm{B}$ ). The earlier researches have also observed the absorption spectrum between 370 and 390 nm [19]; Fesharaki., et al. 2010; Zhang., et al. 2011; Hariharan., et al. 2012).

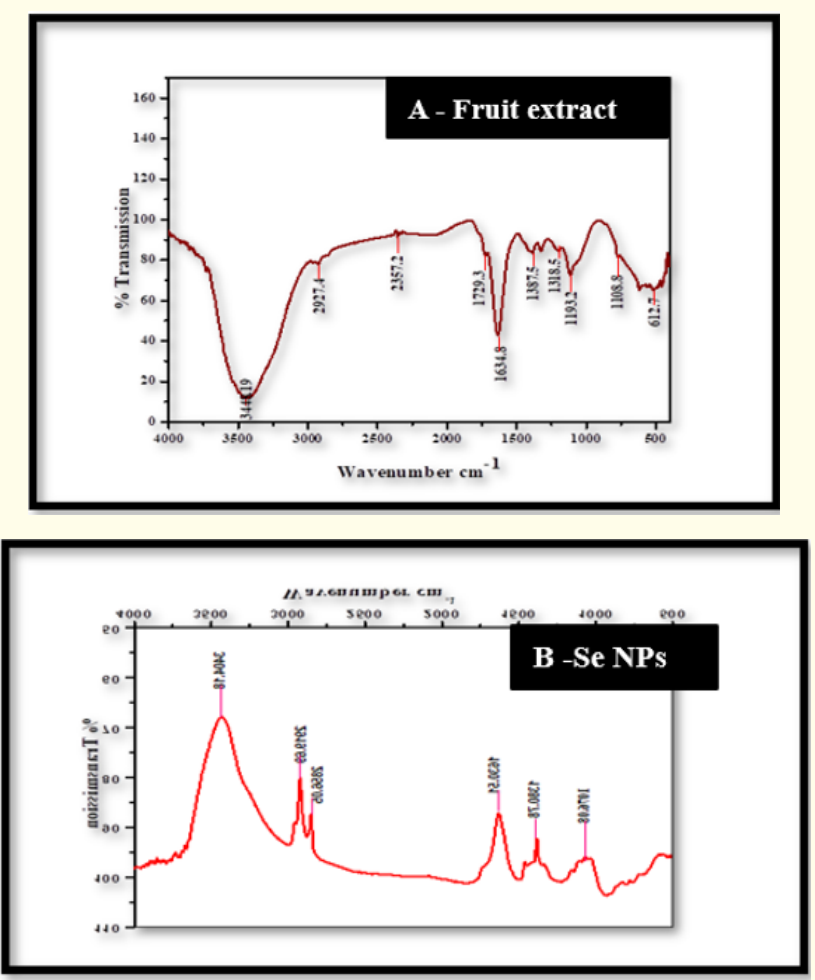

Figure 5: (A) FTIR images showed that fruit extract of M. cymbalaria (B) synthesized Se NPs using fruit extract of M. cymbalaria.
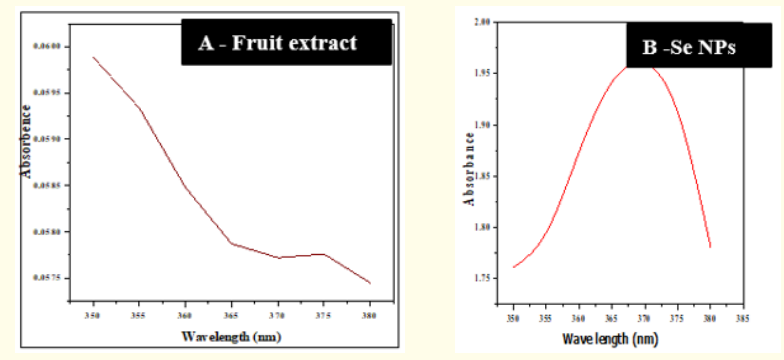

Figure 6: (A) UV results showed that fruit extract of M. cymbalaria

(B) Synthesized Se NPs using fruit extract of M. cymbalaria.

\section{Antibacterial activity}

It is evidence for our study biosynthesized Se NPs had antibacterial activity against all the human pathogenic bacterial strains 
such as Staphylococcus aureus, Bacillus cereus, Escherichia coli, Pseudomonas aeruginosa and Klebsiella pneumonia. The maximum zone of inhibition was formed at $26 \mathrm{~mm}$ at a concentration of 100 $\mathrm{mg} / \mathrm{ml}$ and the minimum zone of inhibition at $8 \mathrm{~mm}$ (Figure 7). The earlier researchers are reported dose dependent antibacterial activities of biosynthesized Se NPs (Boroumand., et al. 2019).

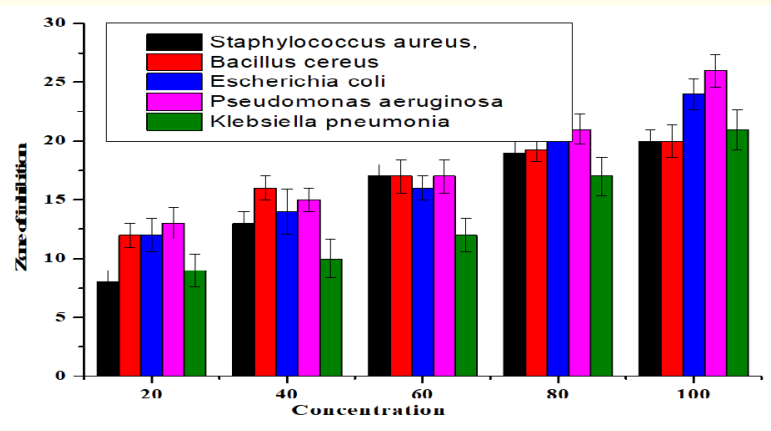

Figure 7: Antibacterial activity of fruit extracts and synthesized Se NPs.

\section{Conclusion}

We conclude that the present study was effective use of $M$. Cymbalaria fruit extract and synthesis of Se NPs. Then the fabricated Se NPs was characterized using UV-VIS, XRD, FTIR, TEM, and SEMEDX. The fabricated Se NPs exhibits a spherical shape with an average diameter between $15-18 \mathrm{~nm}$ and it is confirmed by TEM analysis. The biosynthesized Se NPs assessed the antibacterial activity against human pathogenic bacterial strains. To understand the better mechanism and effects of biosynthesized Se NPs on their potential benefits.

\section{Bibliography}

1. Anastas P T and Warner J C. "Green chemistry" theory and practice”. Oxford university press (2000).

2. Ashokkumar S., et al. "Synthesis of silver nanoparticles using A". Indicum Leaf Extract and their Antibacterial Activity (2015).

3. Boroumand S., et al. "Selenium nanoparticles: synthesis, characterization and study of their cytotoxicity, antioxidant and antibacterial activity". Materials Research Express 6 (2019): $0850 \mathrm{~d} 8 \mathrm{~m}$.
4. Chen T., et al. "Selenium nanoparticles fabricated in Undaria pinnatifida polysaccharide solutions induce mitochondriamediated apoptosis in A375 human melanoma cells". Colloids and Surfaces B: Biointerfaces 67 (2008): 26-31.

5. Cremonini E., et al. "Biogenic selenium nanoparticles: characterization, antimicrobial activity and effects on human dendritic cells and fibroblasts". Microbial Biotechnology 9 (2016): 758-771.

6. Cushing BL., et al. "Recent advances in the liquid-phase syntheses of inorganic nanoparticles". Chemical Reviews 104.9 (2004): 3893-3946.

7. Dorofeev GA., et al. "Determination of nanoparticle sizes by Xray diffraction". Colloid Journal 74 (2012): 675-685.

8. Duran N., et al. "Mechanistic aspects of biosynthesis of silver Nanoparticles by several Fusarium oxysporum strains". Journal of Nanobiotechnology 3.8 (2005).

9. Durán N., et al. "Mechanistic aspects in the biogenic synthesis of extracellular metal nanoparticles by peptides, bacteria, fungi, and plants". Applied Microbiology and Biotechnology 90 (2011): 1609-1624.

10. Fesharaki P J., et al. "Biosynthesis of selenium nanoparticles using Klebsiella pneumoniae and their recovery by a simple sterilization process". Brazilian Journal of Microbiology 41 (2010): 461-466.

11. Gour A and Jain N K. "Advances in green synthesis of nanoparticles". Artificial Cells, Nanomedicine, and Biotechnology 47.1 (2019): 844-851.

12. Hariharan H., et al. "Microbial synthesis of selenium nanocomposite using Saccharomyces cerevisiae and its antimicrobial activity against pathogens causing nosocomial infection". Chalcogenide Letters 9.12 (2012): 509-515.

13. Hu C H., et al. "Comparative effects of nano elemental selenium and sodium selenite on selenium retention in broiler chickens". Animal Feed Science and Technology 177.3-4 (2012): 204-210. 
14. Jha D K., et al. "Medicinal use of an ancient herb Momordica Cymbalaria: a review". International Journal of Pharmaceutical Sciences and Research 9.2 (2018): 432-441.

15. Jiang J., et al. "Characterization of size, surface charge, and agglomeration state of nanoparticle dispersions for toxicological studies". Journal of Nanoparticle Research 11.1 (2009): 77-89.

16. Kargar Razi., et al. "Preparation of nano selenium particles by water solution phase method from industrial dust". International Journal of Nano Dimension 1 (2011): 261-267.

17. Khiralla GM and El-Deeb BA. "Antimicrobial and antibiofilm effects of selenium nanoparticles on some foodborne pathogens". LWT-Food Science and Technology 63.2 (2015): 10011007.

18. Kiruba S., et al. "Therapeutic Uses of Momordica Cymbalaria". Indian Journal of Public Health Research and Development 10.11 (2019).

19. Malhotra S., et al. "A superficial synthesis of selenium nanospheres using wet chemical approach". The International Journal of Nanotechnology and Applications 3 (2014): 7-14.

20. Mallikarjuna K., et al. "D. P. Green synthesis of silver nanoparticles using Ocimum leaf extract and their characterization". Digest Journal of Nanomaterials and Biostructures 6.1 (2011): 181-186.

21. Pal D., et al. "Biological activities and medicinal properties of Cajanus cajan (L) Millsp". Journal of Advanced Pharmaceutical Technology and Research 2.4 (2011): 207.

22. Swamy MK., et al. "Synthesis and characterization of silver nanoparticles using fruit extract of Momordica cymbalaria and assessment of their in vitro antimicrobial, antioxidant and cytotoxicity activities". Spectrochimica Acta Part A: Molecular and Biomolecular Spectroscopy 151 (2015): 939-944.

23. Zhang W., et al. "Biosynthesis and structural characteristics of selenium nanoparticles by Pseudomonas alcaliphila". Colloids and Surfaces B: Biointerfaces 88.1 (2011): 196-201.
Volume 5 Issue 12 December 2021

(C) All rights are reserved by Paulraj Suganya., et al. 